\title{
Simulation of light propagation in photochemical fixed-bed reactors using a BRDF-based model
}

Received 00th January 20xx, Accepted 00th January 20xx DOI: $10.1039 / \times 0 \times x 00000 x$

\author{
Bruno Ramos, ${ }^{\text {aa }}$ Franco Grisafi, ${ }^{\mathrm{b}}$ Alberto Brucato ${ }^{\mathrm{b}}$ and Antonio Carlos Silva Costa Teixeira ${ }^{\mathrm{a}}$
}

Modelling light transport in fixed-bed photochemical reactors can be challenging if the geometry of the packing is the object of investigation. In this manuscript, we present a physically-based model of light transport for the simulation of fixed-bed photochemical reactors to be coupled with explicit consideration of reactor geometry: spatial properties of the fixed bed, such as size, shape, distribution and quality of the surface of packing particles are used as input variables. The existence of a catalytic coating on the packing surface, and its major properties such as spectral coefficient of absorption and surface rugosity can also be easily coupled with the light propagation algorithm. The model was built upon the framework of the bidirectional reflectance distribution function (BRDF), using the microfacets theory (MFT) to evaluate an approximate solution. As an example of application, easily measurable experimental data, such as UV absorption/extinction spectra and surface roughness, and readily available literature data on spectral refractive indices are used as inputs to calculate (i) the fate of the irradiated energy (percentage absorbed, transmitted and scattered-out) and (ii) the spatial distribution of the scattered rays. Taken together, these output data should offer the engineer guidelines for the design of fixed-bed photochemical reactors with optimised light collection and distribution.

\section{Introduction}

Light transport is not conventionally part of the Chemical Engineering classical Transport Phenomena ensemble, within which most problems are sufficiently described by mass, energy and momentum conservation. However, the search for new reaction windows - less reliant on conventional energy sources and somewhat more sustainable - has brought about a need for new chapters on transport phenomena books. In this context, understanding light transport is fundamental to accurately design and optimise equipment for all sorts of technologically relevant photochemical processes, from organic syntheses such as the Claisen rearrangement ${ }^{1}$ or methane reforming ${ }^{2}$ - to photocatalytic water splitting ${ }^{3}$ and the growth of microalgae $e^{4,5}$ It becomes even more important if the equipment to be designed or optimised makes use of directionally and temporally intermittent light sources, such as solar radiation. These "updates" to classical transport phenomena syllabuses are not exclusive to light transport: a couple of years ago, reviews and perspectives published in specialised literature urged that more studies be dedicated to basic transport phenomena that are likely to affect Chemical Engineering in ways that were not yet fully understood at that moment. One example is charge carrier transportation in solids ${ }^{6,7}$ : electrons,

\footnotetext{
a. Research Group in Advanced Oxidation Processes (AdOx), Chemical Systems Engineering Centre, Department of Chemical Engineering, University of Sao Paulo, Brazil.

b. Laboratory for Chemical and Biochemical Plants, Department of Industrial and

Digital Innovation, University of Palermo, Italy.

* Corresponding author: bruno.ramos@usp.br.
}

vacancies; subatomic particles and crystal defects. From the Physics perspective, these phenomena might be reasonably well known; but the role they play in a chemical reaction, the scale of their importance, and how this knowledge can be used to improve existing equipment is yet to be fully pictured.

Most light transport models used in the chemical engineering literature derive from studies on radiative heat transport, from the $1960 \mathrm{~s}^{8}$, in the framework of the Radiative Transport Equation $(\mathrm{RTE})^{9,10}$. The classical RTE incorporates all major phenomena occurring in light propagation: when travelling through an interactive medium, photons can be transmitted, absorbed, scattered (i.e. reflected or refracted) or emitted (e.g. by fluorescence). These phenomena are translated, in terms of wave-physics, into changes of radiation intensity. Many models have been built based on the RTE; each model taking a different approach to the mathematical systematisation of the phenomena. For instance, absorption may be evaluated by a purely ab-initio approach - as a function of the internal energetic structure of the absorbing material (HOMO/LUMO or band energies) and the surrounding electromagnetic fields ${ }^{11}-$, or by relying on experimental data and the well-known BeerLambert relationship. Absorption and emission parameters have clear and simple ways of being evaluated, but the picture is slightly more complex for scattering. The majority of models in current use $\mathrm{e}^{10-14}$ were designed to simulate scattering particles with negligible dimensions and random geometries (such as a suspension or a colloid). While this approach suffices to describe most relevant photocatalytic systems, it is clearly inappropriate to simulate larger systems in which the geometry, the size, the position, and the nature of the interacting objects play a significant role, such as packed bed reactors. 
Mathematically, radiation propagation problems are often treated by discrete-ordinate (DOM) ${ }^{12}$, finite volume (FVM) $)^{9,10}$ or stochastic methods ${ }^{13,14}$. A thorough discussion on these methods can be found elsewhere ${ }^{15}$. Stochastic models allow for exact calculations, but are limited to small systems due to the high CPU costs required ${ }^{16}$; whereas DOM or FVM can be implemented in computational fluid dynamics packages and make good use of well-optimised meshing strategies to save computational time and provide a good approximation of the RTE solution ${ }^{17}$.

The model reported in this contribution is specific to the simulation of bed reactors consisting of packings with specific (and relevant) geometries and a macroscopic size distribution, considering (i) the shape and (ii) size of the particles, and (iii) their surface roughness; (iv) the material of the packing; (v) the presence of a catalytic layer and (vi) its thickness; and, lastly, (vii) the wavelength and (viii) the emission aperture of the light source. As output, the model retrieves (i) the mean path travelled by the photons inside the reactor configuration; (ii) statistics on the fate of the photons, i.e. the percentage of surface absorption, transmission and loss; and (iii) the angular distribution of the outbound photons. Taken together, these data should empower the engineer with guidelines for choosing and distributing an appropriate packing material for maximising photon harnessing.

\section{Model foundations}

The model is described by the algorithm shown in Figure 1. Each photon is initialised and displaced in an initial direction until it reaches a surface. At the surface, the probabilities of reflection, absorption and transmission (refraction) are evaluated using previously gathered data. The new propagation direction is calculated, and the photon is allowed to continue its movement until the next interaction with a surface of the system. The algorithm is based on the following hypotheses:

i. There is no emission of UV-A radiation within the reactor body;

ii. All scattering events are elastic, i.e. energy losses are negligible and the wavelength of the photon is considered constant throughout the propagation pathway;

iii. Photon absorption is a binary event (i.e. a photon cannot be partially absorbed)

iv. Due to the size of the scattering particles, diffraction is neglected.

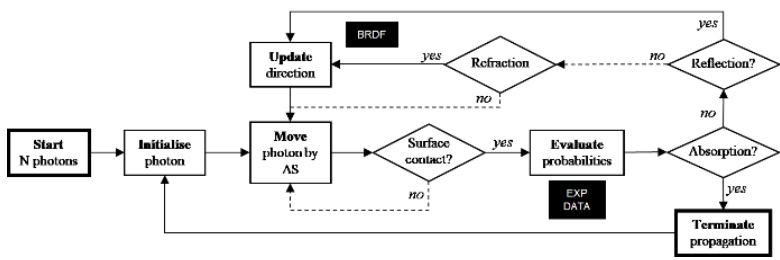

Figure 1. Raytracing algorithm.

\section{Photon Initialisation}

The first step of the algorithm is the definition of the photon initial position and travelling direction. These parameters will depend on the type of light source ${ }^{10,18}$. Sunlight is, at first look, the simplest radiation to model at any given point in time: all rays follow the same direction (parallel emission). Establishing the exact direction and its time-dependent change can be determined by solar-tracking ${ }^{19,20}$ models. Emission from artificial light sources is often separated in two different scenarios: arc lamps, for instance, have a volumetric emission, i.e. the emission centre is located at some fixed point within the lamp volume, and the radiation profile can be safely modelled as an isotropic quantity ${ }^{21}$. Other types of lamps emit radiation from a substance coated on its internal surfaces, such as fluorescent tubes and blacklight lamps; in this case, a surfaceemission model, generally following a Lambertian pattern, is more appropriate 9 .

\section{Surface contact}

The calculation of surface contact points depends on the mathematical definition of the system. System geometry can be considered explicitly, i.e. with the positions and sizes of all elements within the control volume mathematically specified; or implicitly, by treating all solids as exposed faces at random orientations and randomised positions within the modelled volume. At each cycle, given a ray propagation direction and an initial point, the surface contact positions (if explicit) or probabilities (if implicit) are determined by vector algebra.

\section{A probabilistic approach to absorption and transmission}

The probabilities of absorption, $\mathrm{p}(\mathrm{abs})$; reflection, $\mathrm{p}(\mathrm{ref})$; and transmission, $\mathrm{p}$ (trans), are evaluated using spectral data from absorption (absorbance, $A(\lambda, \varepsilon)$ ) and extinction $(E(\lambda, \varepsilon)$ ) analyses, obtained either experimentally or by theoretical simulation 22 , as follows:

$$
\begin{gathered}
p(\text { abs })+p(\text { ref })+p(\text { trans })=1.0 \\
E(\lambda, \varepsilon)=1-T_{e x}(\lambda, \varepsilon)=A(\lambda, \varepsilon)+S(\lambda) \\
A(\lambda, \varepsilon)=1-T_{a b s}(\lambda, \varepsilon)=p(\text { abs }) \\
S(\lambda)=E(\lambda, \varepsilon)-A(\lambda, \varepsilon)=p(\text { ref }) \\
T_{e x}(\lambda, \varepsilon)=1-E(\lambda, \varepsilon)=p(\text { trans })
\end{gathered}
$$

where all probabilities should obey the constraint given by Equation 1 and be evaluated as functions of the irradiation wavelength $(\lambda)$ and, be it the case, the catalytic layer thickness $(\varepsilon)$. For simulations of transparent materials, the probability of reflection is given by the Fresnel equation,

$$
p(\text { ref })=\frac{1}{2}\left(\left|\frac{n_{1} \cos \left(\theta_{i}\right)-n_{2} \cos \left(\theta_{t}\right)}{n_{1} \cos \left(\theta_{i}\right)+n_{2} \cos \left(\theta_{t}\right)}\right|^{2}+\left|\frac{n_{1} \cos \left(\theta_{t}\right)-n_{2} \cos \left(\theta_{i}\right)}{n_{1} \cos \left(\theta_{t}\right)+n_{2} \cos \left(\theta_{i}\right)}\right|^{2}\right),
$$


as a function of the incoming $\left(\theta_{i}\right)$ and the transmitted $\left(\theta_{t}\right)$ ray directions, and the refractive indices $\mathrm{n}_{1}$ and $\mathrm{n}_{2}$ of the propagating media. Following Equation 1, the probability of transmittance will be estimated from calculated $p$ (ref) and data on $p$ (abs). To determine the fate of the photon, a random number $\eta_{1} \in[0,1]$ is generated and compared against $p(a b s)$ :

$$
\eta_{1} \leq p(a b s)=\text { absorption }
$$

else, another number $\eta_{2} \in[0,1]$ is generated and compared against $p($ ref):

$$
\eta_{2} \leq p(\text { ref })=\text { reflection }
$$

In case both absorption and reflection tests result negative, the photon is transmitted through the interface, with a new propagation direction assigned based on the Snell's Law. If the photon is absorbed, propagation is terminated and a new photon is generated. If the photon is reflected, the BRDF-based model is called to determine the new propagation direction.

\section{Reflection and the BRDF}

The bidirectional reflectance distribution function (BRDF) is a mathematical description of how photons are reflected from an opaque surface. It was first defined in the 1960s by Fred Nicodemus ${ }^{23}$, motivated by the lack of directional elements of emitting or reflecting opaque surfaces (i.e. their macroscopic curvature) in the mathematical description of their reflectance or emissivity. In this paradigm, the reflectance $\rho$ of a surface element is given as a four-dimensional function representing the fraction of the light irradiated from a direction $\omega_{i}$ that is radiated away in the outgoing direction $\omega_{\circ}$ :

$$
\rho\left(\omega_{i}, \omega_{o}\right)=\frac{\partial L\left(\omega_{o}\right)}{\partial I\left(\omega_{i}\right)}=\frac{\partial L\left(\omega_{o}\right)}{L\left(\omega_{i}\right)\left\langle\omega_{i}, \omega_{n}\right\rangle \partial \omega_{i}}
$$

where $\omega$, the incoming (i), outgoing (o) or surface-normal (n) direction, is best described by the spherical angles $\vartheta$ and $\varphi$. $L$ and $/$ denote the radiance $\left[\mathrm{W} \mathrm{m}^{-2} \mathrm{sr}^{-1}\right]$ and the irradiance $[\mathrm{W} \mathrm{m}$ $\left.{ }^{2}\right]$, respectively, at the specific point on the surface where reflection takes place. There are several approaches to solving the BRDF, varying according to the degree of accuracy, the materials/geometries on which they are to be applied, and their reliance on previously gathered experimental data. In this model, we opted to use the approach known as the micro-facets theory, developed by Cook and Torrance ${ }^{24}$ in the 1980 s for computer graphics (CG), given its flexibility to model different materials and the wide acceptance and use it has received in the CG community.

According to the micro-facets approach, any microscopic element of a macroscopic geometry $G$ can be described as a micro-surface $M$ characterised by a collection of points $x_{m}$ with a respective normal direction $\omega_{m}$ (Figure 2). The micro-facet model is thus a statistical model of the optical properties of this micro-surface. The first step to describe the micro-surface is a definition of the distribution of normals, i.e. the statistical distribution of the possible surface-normal unit vectors, $D\left(\omega_{m}\right)$. To evaluate this distribution, it is necessary to have a topographic description of the surface being modelled. From this topographic description, a suitable distribution of normals is determined. In this work, we have used the $\mathrm{GGX}^{25}$ distribution, given by Equation 10,

$$
D\left(\omega_{m}\right)=\frac{\chi^{+}\left(\omega_{m} \cdot \omega_{g}\right)}{\pi \alpha^{2} \cos ^{4} \theta_{m}\left(1+\frac{\tan ^{2} \theta_{m}}{\alpha^{2}}\right)},
$$

as a function of $\alpha$, the rugosity, evaluated from the average RMS of slopes $a$ of the distribution and each particular outbound direction $\theta_{\mathrm{o}}$,

$$
\alpha=\frac{1}{a \tan \left(\theta_{o}\right)}
$$

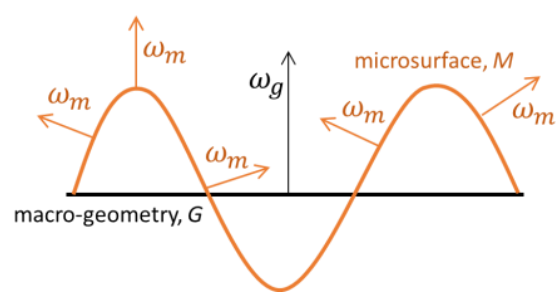

Figure 2. Micro-surface representation of a macro-scale surface-element

With an expression for the $D\left(\omega_{m}\right)$, the BRDF (Equation 9) can be rewritten as:

$$
\begin{aligned}
& \rho\left(\omega_{o}, \omega_{i}\right)=\frac{d L\left(\omega_{o}, M\right)}{\cos \left(\theta_{i}\right) L\left(\omega_{i}\right) d \omega_{i}} \\
& =\frac{1}{\cos \left(\theta_{i}\right)} \int \rho_{M}\left(\omega_{o}, \omega_{i}, \omega_{m}\right)\left\langle\omega_{i}, \omega_{m}\right\rangle D_{o}\left(\omega_{m}\right) d \omega_{m}
\end{aligned}
$$

or, expanding $D_{o}$ - the distribution of visible normals, i.e. of normals faced towards an outbound direction $\omega_{o}$ :

$$
\begin{gathered}
\rho\left(\omega_{o}, \omega_{i}\right)=\frac{1}{\cos \left(\theta_{o}\right) \cos \left(\theta_{i}\right)} \times \\
\int \rho_{M}\left(\omega_{o}, \omega_{i}, \omega_{m}\right)\left\langle\omega_{o}, \omega_{m}\right\rangle\left\langle\omega_{i}, \omega_{m}\right\rangle G_{2}\left(\omega_{i,} \omega_{o}, \omega_{m}\right) D\left(\omega_{m}\right) d \omega_{m},
\end{gathered}
$$

where $G_{2}$ is a function accounting for the shadowing and masking effects that the elements of surface topology exert on the observed reflectance (Figure $\mathbf{3}$ ).

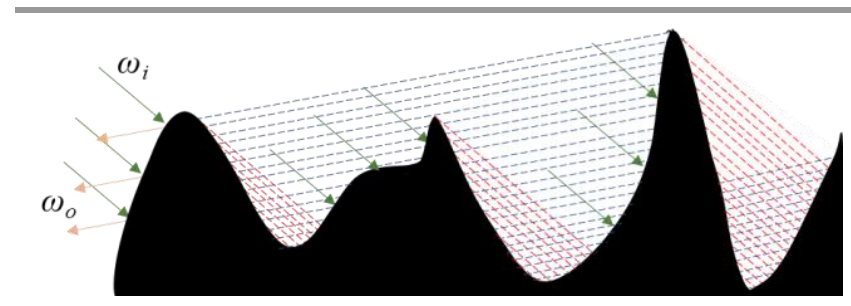

Figure 3. Illustration of the shadowing (red dotted area) and masking (blue dotted area) effects promoted by a rough surface, for a pair of inbound (green arrows) and outbound (orange arrows) directions $\left(\omega_{i}, \omega_{0}\right)$.

Each micro-facet is considered to have specular and diffuse reflectance. In the former case, for a perfect mirror, the reflectance of each micro-facet with normal $\omega_{m}$ is expressed as $^{25}$

$$
\rho_{M, S}\left(\omega_{o}, \omega_{i}, \omega_{m}\right)=\frac{F\left(\omega_{o}, \omega_{h}\right) \delta_{w_{h}}\left(\omega_{m}\right)}{4\left|\omega_{i} \cdot \omega_{h}\right|^{2}},
$$


where $\omega_{h}$ is the half-direction between $\omega_{i}$ and $\omega_{o}$, and $F\left(\omega_{o}, \omega_{h}\right)$ is the Fresnel reflectance, evaluated in this work by the less computationally-demanding Schlick's approximation ${ }^{26}$ :

$$
\begin{gathered}
F\left(\omega_{o}, \omega_{h}\right)=F\left(\omega_{h}\right)=F_{m}+\left(1-F_{m}\right)\left(1-\cos \left(\theta_{h}\right)\right)^{5} \\
F_{m}=\left|\frac{n_{1}-n_{2}}{n_{1}+n_{2}}\right|^{2}
\end{gathered}
$$

The purely diffuse reflectance is given for each micro-facet simply by Equation 17:

$$
\rho_{M, d}\left(\omega_{o}, \omega_{i}, \omega_{m}\right)=\frac{1}{\pi}
$$

The Dirac distribution in Equation 14 allows for the substitution of the integral in Equation 12 by its integrand evaluated at $\omega_{\mathrm{m}}$ $=\omega_{h}$. Additionally, considering that $\left|\omega_{i} \cdot \omega_{h}\right|=\left|\omega_{o} \cdot \omega_{h}\right|$, it is possible to simplify the macroscopic specular BRDF as:

$$
\rho_{s}\left(\omega_{o}, \omega_{i}\right)=\frac{F\left(\omega_{o}, \omega_{h}\right) G_{2}\left(\omega_{o}, \omega_{i}\right) D\left(\omega_{h}\right)}{4\left|\omega_{g} \cdot \omega_{o}\right|\left|\omega_{g} \cdot \omega_{i}\right|}
$$

The macroscopic diffuse BRDF instead does not have a clear analytical solution. In this work, the following approximation ${ }^{27}$ is used:

$$
\begin{aligned}
\rho_{d}\left(\omega_{o}, \omega_{i}\right)=\left(\frac{1.05}{\pi}\right) & \left(1-F\left(\omega_{o}, \omega_{i}\right)\right) \\
\times & \left(1-\left(1-\left|\omega_{g} \cdot \omega_{o}\right|\right)^{5}\right)
\end{aligned}
$$

The shadowing and masking function $\mathrm{G} 2$ is approximated by 25,28 .

$$
\begin{gathered}
G_{2}\left(\omega_{o}, \omega_{i}\right)=G_{1}\left(\omega_{o}\right) G_{1}\left(\omega_{i}\right) \\
G_{1}(\omega)=\frac{1}{1+\Lambda(\omega)}=\frac{2}{1+\sqrt{1+a^{2} \tan ^{2} \theta_{o}}}
\end{gathered}
$$

These equations can be implemented in any raytracing algorithm as a separate function called when a reflection is detected. To evaluate the probabilities of a ray being reflected in a direction $\omega_{0}$, the total reflectance was integrated with respect to each directional angle $\left(\theta_{\circ}\right.$ and $\left.\phi_{0}\right)$ and normalised as a probability distribution function to be sampled:

$$
\begin{aligned}
& p\left(\theta_{o}\right)=\frac{\int \rho\left(\theta_{o}, \phi_{o}\right) d \phi_{o}}{\iint \rho\left(\theta_{o}, \phi_{o}\right) d \theta_{o} d \phi_{o}} \\
& p\left(\phi_{o}\right)=\frac{\int \rho\left(\theta_{o}, \phi_{o}\right) d \theta_{o}}{\iint \rho\left(\theta_{o}, \phi_{o}\right) d \theta_{o} d \phi_{o}}
\end{aligned}
$$

\section{Implementation and simulation of irradiated packed bed reactors}

The algorithm shown in Figure 1, within the framework laid out in the previous section, was implemented in MATLAB (R2017b, MathWorks). Several reactors with fixed spherical packings and varied packing size and material (glass, unpolished alumina, polished iron) were simulated using a code developed to construct the geometry of a cylindrical tube with a random packing of equally-sized spheres. This was carried out by modifying an approach used for solving the so-called equal circles packing problems (ECPP) ${ }^{29,30}$, which consisted of making an initial guess based on analytical geometry and treating the inner circles as elastic objects, thereby assigning the system a total elastic energy content that can be minimised with respect to the positions of the circles in relation to each other and in relation to the outer circle. The model was adapted to represent a three-dimensional system, and a code was written as to provide a $\mathrm{n} \times 3$ matrix containing the optimal positions of the centres of the $\mathrm{n}$ spheres simulated, using the software's built-in minimisation with constraints algorithm (fmincon). An initial guess is generated as a function of the radius $r_{p}$ of the spheres, and the inner radius $R$ of the reactor's external case, using simple geometry. Two studies were carried out to investigate light absorption and distribution inside the reactors: one regarding the light-source emission pattern; and the other regarding the aspect ratio $\mathrm{R} / \mathrm{r}$ of the packed-bed reactor, and will be presented next.

\section{Measurement of the rugosity parameter}

The parameter $a$ in Equations 11 and 21, representing the surface RMS roughness, can be estimated by local scan electron microscopy (SEM) measurements. Figure 4 illustrates the procedure we adopted for the surface of unpolished alumina beads.
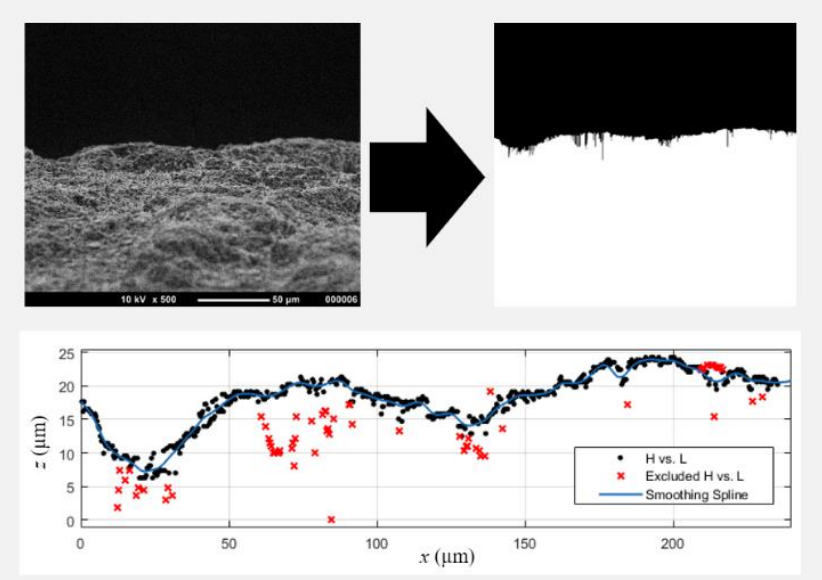

Figure 4. Illustration of the analysis of RMS slope roughness for the surface of an unpolished alumina microsphere $\left(a=0.29 \mu^{-1}\right)$.

Micrographs of different sections of the surface were analysed in an image processor to obtain surface curves which were subjected to a smoothing filter. In this work, a 5th order Savitzky-Golay smoothing filter was used, as recommended by a white paper of the American Society of Mechanical Engineers for rugosity analysis (ASME B46.1). With this filter, the RMS roughness is given by:

$$
a=\sqrt{\frac{1}{N} \sum_{i=1}^{N} \Delta_{i}^{2}},
$$


where each smoothed data point, $\Delta_{i}$, is evaluated using the filter and the coordinates of identified experimental points as:

$$
\Delta_{i}=\frac{1}{60 \Delta x}\left(y_{i+3}-9 y_{i+2}+45 y_{i+1}-45 y_{i-1}+9 y_{i-2}-y_{i-3}\right)
$$

\section{Raytracing of packed beds with diverse packing materials using the BRDF model: parallel irradiation}

Figure 5 shows some sample raytracing results of reactors with aspect ratio $R / r=5.0$ packed with spherical beads made of (a) borosilicate glass and (b) alumina, irradiated from the top by a parallel light source (e.g. sunlight), and coated with a thin $\mathrm{TiO}_{2}$ layer. Table 1 summarises simulation results considering the destination of irradiated photons for both uncoated (i.e. without catalyst) packings, and for packings covered with a thin $(6 \mu \mathrm{m})$ photocatalytic layer composed of mixed-phase $\mathrm{TiO}_{2}$. In a scenario without the catalyst, photons could be either reflected out of the reactor or transmitted through it after several scattering events. When the catalyst is present, absorption was an additional possibility. Table 1 also reports the simulation time per ray, the number of redirections (by either refraction or reflection), and the length of the path it traversed through the system, as a percentage of the reactor radius.

The results indicate that by replacing coated glass beads with alumina beads, the amount of energy absorbed should be reduced by ca. $39 \%$, whereas the average path length traversed by the rays should be increased by $16 \%$. Considered together with the higher number of reflections, the simulation suggests that $\mathrm{Al}_{2} \mathrm{O}_{3}$ provides an improved distribution of light inside the reactor, which might result in a larger reaction space, provided that the unabsorbed photons are redirected within the reactor. Figure $5(\mathrm{~b})$ shows raytracing results for coated $\mathrm{Al}_{2} \mathrm{O}_{3}$ spheres that corroborate this interpretation. A rather broad axial distribution of the rays can be observed clearly in the $\mathrm{Al}_{2} \mathrm{O}_{3}$ packed reactor. Figure 6 shows the angular distribution (with respect to the $x y$-plane) of the rays exiting the reactor. The higher specular reflectivity of iron-determined in the model by its small surface roughness $(a<0.01)$ - can be seen by the peak reflection around $\vartheta=90^{\circ}$. Because of its rough surface, corresponding to an increased contribution of diffuse reflectivity to the overall reflectance, $\mathrm{Al}_{2} \mathrm{O}_{3}$ exhibits a broader angular distribution. Glass, being highly transparent to the selected wavelength, sees the largest number of rays being collected out in the hemisphere opposite to the light-source $(\vartheta$ $>180^{\circ}$ ).
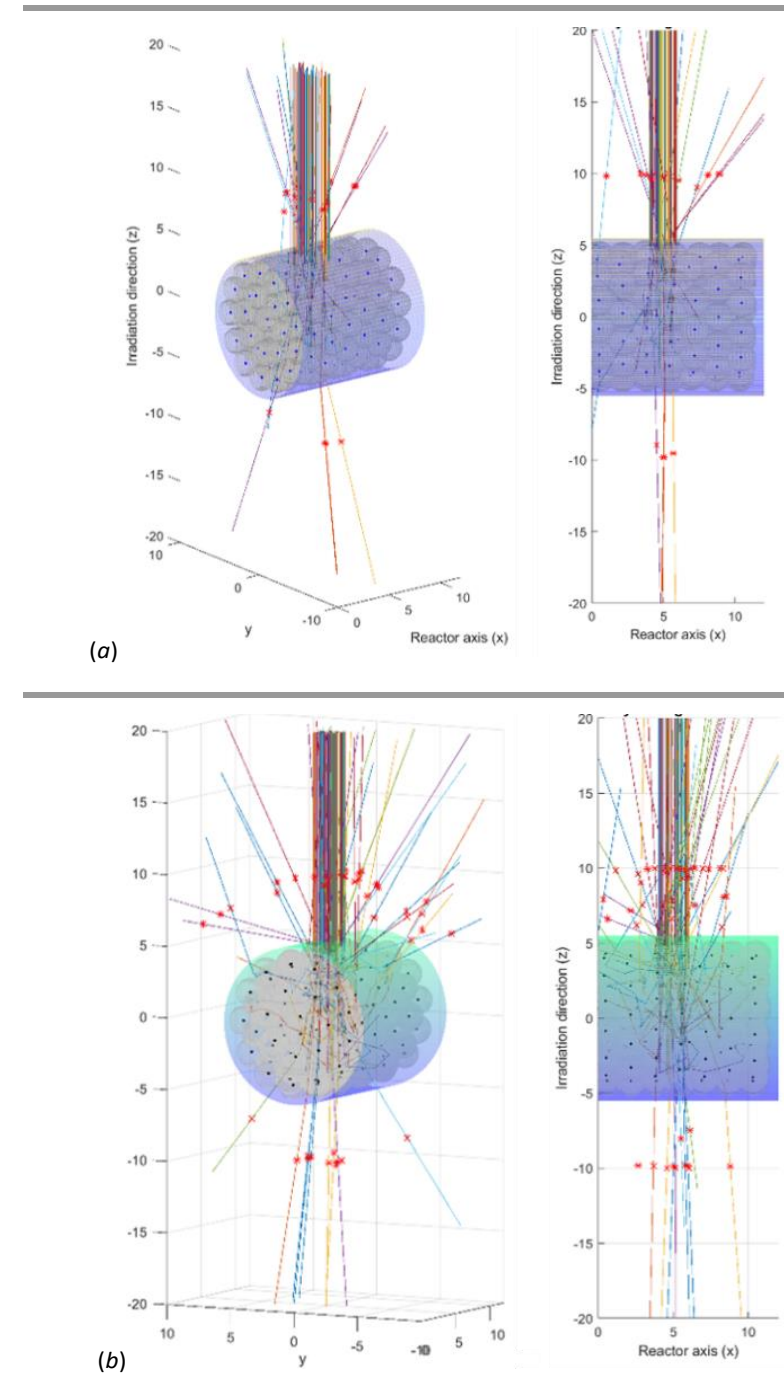

Figure 5. Raytracing results for PBR with aspect ratio $R / r=5$, illuminated by a parallel light source and packed with $\mathrm{TiO}_{2}$-coated $(a)$ glass and $(b) \mathrm{Al}_{2} \mathrm{O}_{3}$ spheres.

Table 1. Simulation results (1,000 rays) for a packed-bed reactor $\left(\mathrm{R} / \mathrm{r}=5, A_{\text {irrad }}=4.03 \mathrm{~cm}^{2}\right)$ irradiated by a monochromatic ( $\left.365 \mathrm{~nm}\right)$ parallel light-source.

\begin{tabular}{ccccc}
\hline Packing & $\begin{array}{c}\text { Simulation time } \\
\text { (s/ray) }\end{array}$ & $\begin{array}{c}\text { Average number of } \\
\text { redirections }\end{array}$ & Reflections (\%) & Photons absorbed (\%) \\
\hline Uncoated glass & 1.83 & 9.8 & 18.4 & 0.00 \\
$\mathrm{TiO}_{2}$-coated glass & 0.44 & 4.6 & 13.0 & 79.3 \\
Uncoated $\mathrm{Al}_{2} \mathrm{O}_{3}$ & 0.73 & 5.8 & 61.0 & 0.00 \\
$\mathrm{TiO}_{2}$-coated $\mathrm{Al}_{2} \mathrm{O}_{3}$ & 0.60 & 3.8 & 43.4 & 0.74 \\
Uncoated $\mathrm{Fe}$ & 0.69 & 3.7 & 98.4 & 0.64 \\
$\mathrm{TiO}_{2}$-coated $\mathrm{Fe}$ & 0.63 & 3.8 & 42.3 & 0.00 \\
\end{tabular}




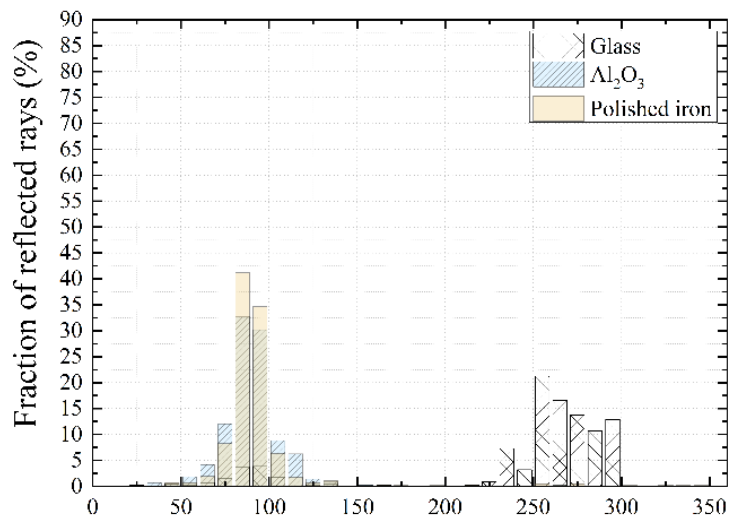

(a)

Reflected angle (deg)

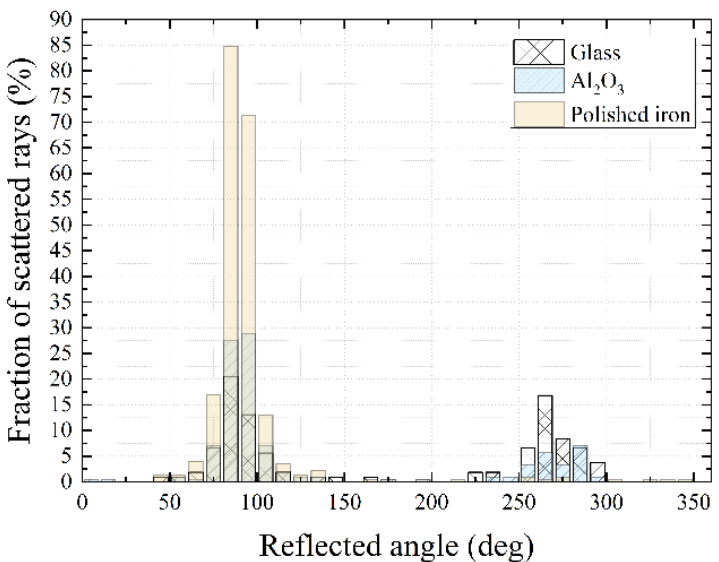

(b) Figure 6. Distribution of exiting ray directions for each packing material, $(a)$
uncoated and $(b)$ coated with a $6-\mu \mathrm{m}$ layer of $\mathrm{TiO}_{2}$. (Parallel irradiation, $\mathrm{R} / \mathrm{r}=$
5 )

Raytracing of packed beds with diverse packing materials using the BRDF model: point source

If the light source is replaced by a point source, such as an LED array, the destination of the photons as well as their distribution is expected to change, as shown in Table 2 and Figure 7. These simulations were carried out for a source with emission aperture of 10 degrees, considering the same emission area as the previous simulations with a parallel light source, positioned $20 \mathrm{~cm}$ above the reactor walls, resulting in an external irradiated area $A_{\text {irrad }}$ of $83.95 \mathrm{~cm}^{2}$.

The increased aperture means that the irradiated area $A_{\text {irrad }}$ is substantially increased with respect to the parallel irradiation.
In fact, for the conditions of the simulation, $A_{\text {irrad }}$ saw a 20 -fold increase. Thus, the incoming rays are expected to spread through a larger volume inside the reactor, interacting with more beads and increasing their probability of absorption. A comparison of the results shown in Figures 7 and 8 reinforces the fact that light from point sources is scattered in broader distributions, for all opaque, transparent and specular materials. Absorption calculations suggest that using a diffuse scatterer, such as alumina, might provide improved photon harnessing when changing from a parallel to a point light source (ca. 3.5\%); whereas the absorption efficiency decreases sharply using specular materials when promoting the same change in illumination pattern.

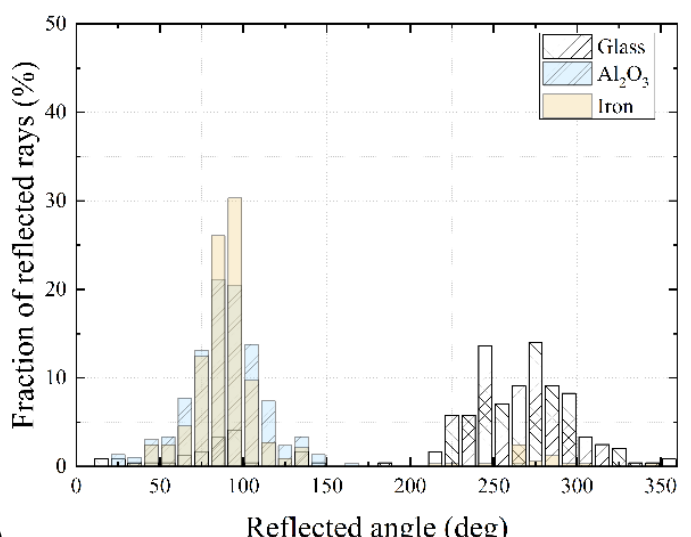

(a)

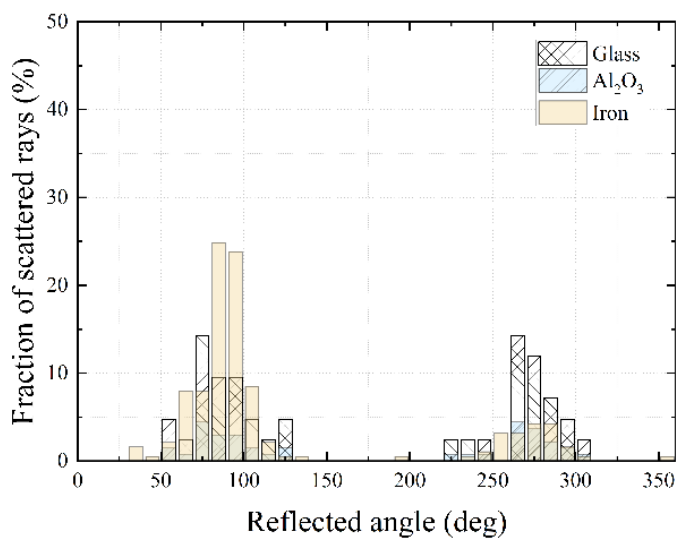

Figure 7. Distribution of exiting ray directions for each packing material, (a) uncoated and (b) coated with a $6 \mu \mathrm{m}$ layer of TiO2. (Point-source, $R / r=5$ )

Table 2. Simulation results (1,000 rays) for a packed-bed reactor $\left(\mathrm{R} / \mathrm{r}=5, A_{\text {irrad }}=83.95 \mathrm{~cm}^{2}\right)$ irradiated by a monochromatic ( $\left.365 \mathrm{~nm}\right)$ point source.

\begin{tabular}{|c|c|c|c|c|c|}
\hline Packing & Simulation time (s/ray) & $\begin{array}{l}\text { Average number of } \\
\text { redirections }\end{array}$ & Reflections (\%) & $\begin{array}{c}\text { Photons } \\
\text { absorbed (\%) }\end{array}$ & Path length (\% R) \\
\hline Uncoated glass & 1.92 & 9.8 & 18.2 & 0.00 & 2.20 \\
\hline $\mathrm{TiO}_{2}$-coated glass & 0.46 & 4.7 & 11.7 & 82.0 & 0.55 \\
\hline Uncoated $\mathrm{Al}_{2} \mathrm{O}_{3}$ & 0.83 & 5.6 & 98.9 & 0.00 & 0.78 \\
\hline $\mathrm{TiO}_{2}$-coated $\mathrm{Al}_{2} \mathrm{O}_{3}$ & 0.65 & 3.5 & 41.8 & 50.1 & 0.74 \\
\hline Uncoated Fe & 0.36 & 2.9 & 99.7 & 0.00 & 0.41 \\
\hline $\mathrm{TiO}_{2}$-coated $\mathrm{Fe}$ & 0.62 & 3.7 & 45.8 & 45.9 & 0.84 \\
\hline
\end{tabular}




\section{Raytracing of packed beds with diverse packing size}

PBRs with varied aspect ratios, for packing radius varying from 0.5 to 3.0, were designed and simulated using the algorithm presented in Figure 1. The outer tube radius $\mathrm{R}$ was chosen to represent an experimental system available in our research group described elsewhere ${ }^{31}$.

Figure 8 shows the absorbed fraction, as a function of the packing radius and material, for parallel (S) and point (L) sources. The results suggest the existence of an optimal packing radius retrieving the highest possible harnessing of the incoming light, around 0.75 and $1.5 \mathrm{~mm}$ for reactors with inner radius $8.8 \mathrm{~mm}$ (corresponding to aspect ratios between 5.9 and 11.7). This effect seems to be more pronounced when opaque packings are used (triangle and circle markers in the figure). This can be understood as a consequence of the reduction in the bed void fraction, increasing, therefore, the amount of radiation scattered out of the reaction volume when smaller packings are used. Larger packings, on the other hand, occupy a larger volume inside the reactor, increasing the probability of light being reflected outwards.

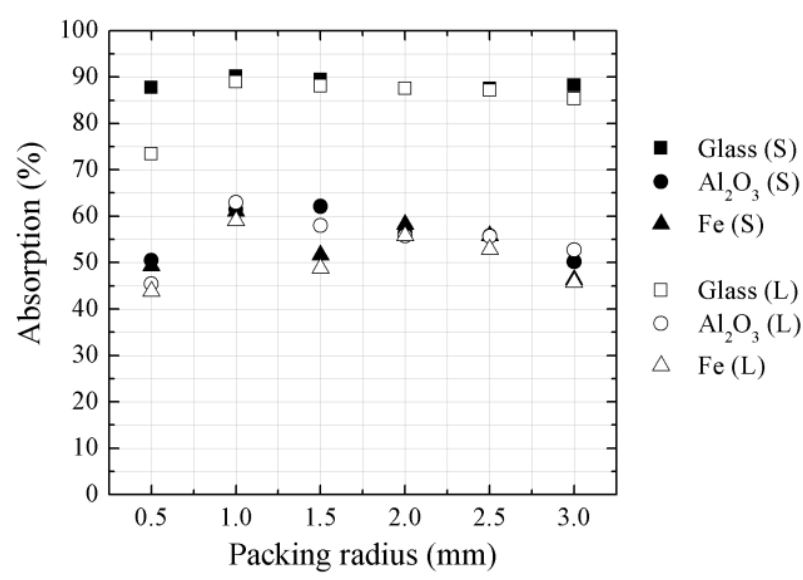

Figure 8. Fraction of incoming light absorbed, as a function of the packing radius for a parallel (S) and a point (L) light source.

\section{Evaluation against experimental data}

Experimental. To evaluate the model performance, we applied it to estimate the yield of salicylic acid in packed-bed photochemical reactors. In aqueous media, benzoic acid is oxidised to salicylic acid by hydroxyl radicals generated locally by a photochemical reaction of nitrite ions with water under UV-A irradiation ${ }^{32}$. Thus, both light absorption and the following chemical reactions happen in the bulk of the flowing medium, not in the surfaces. This configuration was chosen for simplicity: bulk reactions tend to be simpler than surface reactions, where important steps, such as adsorption and desorption, require additional modelling work far beyond the scope of this manuscript. The experimental apparatus, described in detail elsewhere ${ }^{31}$, consists of a tubular reactor (external diameter of $20 \mathrm{~mm}$ ) packed with glass beads of diameters $1.0 \mathrm{~mm}, 3.0 \mathrm{~mm}$ and $6.0 \mathrm{~mm}$. The reactor is irradiated externally by a $100 \mathrm{~W}$ UVA LED chip with emission area of $20 \times 20 \mathrm{~mm}$ and an aperture of
60 degrees, positioned $5 \mathrm{~cm}$ above the reactor external walls. The reaction system consists of $200 \mathrm{~mL}$ of an aqueous solution of sodium nitrite $(1.0 \mathrm{mM})$ - the absorber species -, benzoic acid $(1.0 \mathrm{mM})$ and sodium bicarbonate $(2.5 \mathrm{mM})$, which recirculates between the reactor and a mixing tank at a rate of $33 \mathrm{~mL} \mathrm{~min}-1$ through a peristaltic pump. The oxidation of benzoic acid produces (among others) salicylic acid, which was chosen as tracer and quantified by liquid chromatography using fluorescence detection ${ }^{33}$. The irradiance on the outer walls of the reactor was continuously monitored by a UV sensor. Concentration data were taken and integrated over 120 minutes of reaction. The apparent yield was evaluated from the accumulated concentration of salicylic acid and the accumulated inflow of photons (i.e. mol of product per einstein irradiated).

Model. The extinction coefficients for nitrite ions and quantum yields of formation of salicylic acid are known for a range of photonic energies ${ }^{34,35}$ and were input to the model to simulate light absorption and formation of salicylic acid under each experimental condition. The algorithm shown in Figure 1 was slightly modified to include absorption by the media. Simulations of 1000 rays were repeated 100 times to increase the number of samples, due to the statistical nature of Monte Carlo modelling and the randomness of the reactor packing. The counts of salicylic acid formed were added up and divided by the total amount of rays in the simulation to evaluate the apparent quantum yield; i.e. the amount of product formed per photon irradiated. For comparison purposes, results using two of the most commonly used models in the simulation of light scattering in photochemical reaction engineering, the two-flux (TFM) and six-flux (SFM) approximations, are presented together with the code developed in this work. Both models simplify scattering by lumping the scattered energy into direction bins: TFM assumes that all incident light is either scattered forward or backward ${ }^{36}$, while SFM assumes that, in addition to forward and backward scattering, side scattering (in four directions) is also possible ${ }^{37}$.

Figure 9 shows a comparison between the experimental data and the data obtained from simulations. The experiments show that the yield increases with packing size until an apparent maximum at about $1.5-\mathrm{mm}$ packing radius, and then decreases for further increments in size. This suggests the existence of a region of optimal packing size. Simulations using the BRDF code showed a similar tendency, despite quantitative differences. In contrast, the flux models (SFM and TFM) failed to reproduce this trend, predicting a logarithmic decrease of the yield with respect to the packing radius instead. In quantitative terms, all models seem to predict the range of experimental data with acceptable accuracy, given the complexity of the system. The RMSD of the BRDF code $\left(2.48 \times 10^{-4}\right)$ was found to be about half of the RMSD of the flux models $\left(5.10 \times 10^{-4}\right.$ and $5.31 \times 10^{-4}$ for TFM and SFM respectively), evidencing its superior capability at describing light interactions with a macroscopic heterogeneous system over the latter, simpler models. 


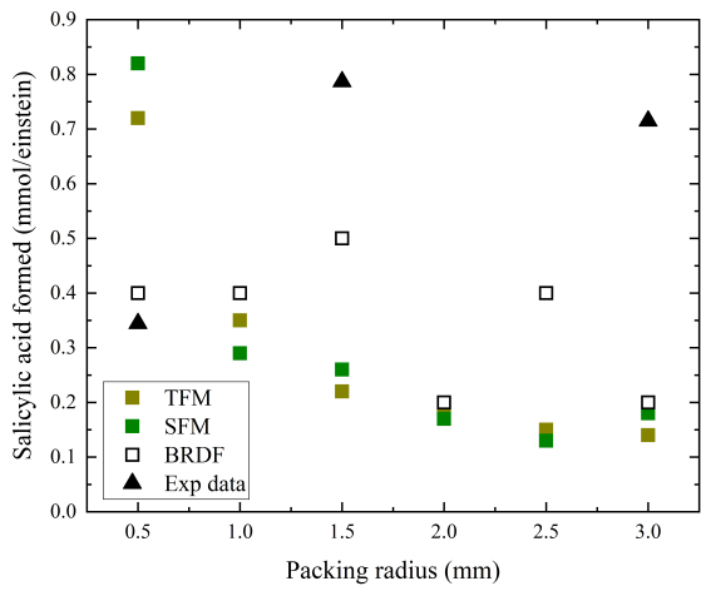

Figure 9. Apparent quantum yield of formation of salicylic acid in tubular packed-bed reactors with various packing sizes. Predictions from simulations
are presented as square symbols, and experimental data are shown as black triangles.

\section{Conclusions}

The model herein reported offers a physically-based mathematical approach to simulate photochemical reactors with specific and explicit geometries for first principles. The main objectives of the BRDF model developed in this work were being sensitive to size, geometry and type of material that makes up the packing of a tubular reactor, a limitation of current, simpler models. In this sense, the model can offer good qualitative predictions of the fate of the incoming photons in a highly scattering system, while also providing statistics regarding the final direction of the reflected radiation - possible guidelines for the design of specific collecting systems.

\section{Conflicts of interest}

There are no conflicts to declare.

\section{Acknowledgements}

The authors would like to acknowledge the support provided by the Sao Paulo Research Foundation (grants \#2017/11583-8 and \#2015/21421-0). Author Ramos would also like to thank the warm reception and the hot coffees provided by the host research group at the University of Palermo.

\section{List of symbols}

$\begin{array}{ll}\text { Symbol } & \text { Definition } \\ \varepsilon & \text { Thickness } \\ \delta & \text { Dirac distribution } \\ \theta_{i} & \text { Incoming angle (wrt normal) } \\ \theta_{t} & \text { Transmitted angle (wrt normal) } \\ \rho_{M, S} & \text { Specular approximation for a } \\ \rho_{M} & \text { micro-facet BRDF (Eq. 14) } \\ & \text { BRDF of a micro-facet }\end{array}$

Dimensions

[L]

$[\angle]$

$[\angle]$

$\left[\mathrm{SR}^{-1}\right]$

$\left[\mathrm{SR}^{-1}\right]$ $\rho_{d} \quad$ Diffuse approximation for a macroscopic BRDF (Eq. 19)

$\chi^{+}(k) \quad$ Heaviside function: 1 if $k>0$ and 0 otherwise

$\omega_{h} \quad$ Half-direction between inbound and outbound directions

RMS roughness (Eq. 24)

Wavelength

Bidirectional reflectance

$[\mathrm{L}]$

$\left[\mathrm{SR}^{-1}\right]$

distribution function (BDRF)

Azimuthal angle (spherical

$[\angle]$

coordinate system)

$\omega_{g} \quad$ Normal direction (macroscopic surface $\mathrm{G}$ )

Incoming direction

$\omega_{m}$

ormal direction (micro-surface M)

\begin{tabular}{|c|c|}
\hline$\omega_{n}$ & Surface normal direction \\
\hline$\omega_{o}$ & Outgoing direction \\
\hline$\alpha$ & Rugosity parameter \\
\hline $\mathrm{A}(\lambda, \varepsilon)$ & Absorbance \\
\hline$D\left(\omega_{m}\right)$ & $\begin{array}{l}\text { Distribution of micro-surface } \\
\text { normals }\end{array}$ \\
\hline$E(\lambda, \varepsilon)$ & Extinction \\
\hline $\mathrm{F}$ & $\begin{array}{l}\text { Fresnel reflectance, } \\
\text { approximated by Eq. } 15\end{array}$ \\
\hline $\mathrm{F}_{\mathrm{m}}$ & $\begin{array}{l}\text { Fresnel reflectance for normal } \\
\text { incidence (Eq. 16) }\end{array}$ \\
\hline $\mathrm{G}_{1}$ & $\begin{array}{l}\text { Smith's shadowing function (Eq. } \\
\text { 21) }\end{array}$ \\
\hline $\mathrm{G}_{2}$ & $\begin{array}{l}\text { Shadowing and masking } \\
\text { function }\end{array}$ \\
\hline I & $\begin{array}{l}\text { Irradiance: radiant flux received } \\
\text { by a surface }\end{array}$ \\
\hline$L$ & $\begin{array}{l}\text { Radiance: radiant flux } \\
\text { emitted/reflected by a surface }\end{array}$ \\
\hline$n$ & Refractive index \\
\hline $\mathrm{p}$ & Probability \\
\hline$S(\lambda)$ & Scattering \\
\hline $\mathrm{T}(\lambda, \varepsilon)$ & Transmittance \\
\hline$x_{m}$ & Point on a micro-surface $M$ \\
\hline
\end{tabular}

\section{References}

F. Galindo, The photochemical rearrangement of aromatic ethers: A review of the Photo-Claisen reaction, $J$. Photochem. Photobiol. C Photochem. Rev., 2005, 6, 123138.

H. Pennemann, R. Bellinghausen, T. Westermann and L. Mleczko, Reforming of Methane in a Multistage Microstructured Reactor, Chem. Eng. Technol., 2015, 38, 1883-1893.

S. S. K. Ma, T. H. Isatomi and K. D. Omen, Hydrogen Production by Photocatalytic Water Splitting, 2013, 56, 280-287. M. Zhao, Microalgae cultivation and metabolites

8 | Manuscript, 2019, 00, 1-9 
production: a comprehensive review, Biofuels, Bioprod. Biorefining, 2018, 12, 304-324. J. K. Pittman, A. P. Dean and O. Osundeko, The potential of sustainable algal biofuel production using wastewater resources., Bioresour. Technol., 2011, 102, 17-25. J. Tyczkowski, Charge Carrier Transfer: A Neglected Process in Chemical Engineering, Ind. Eng. Chem. Res., 2010, 49, 9565-9579. W. J. Ong, L. K. Putri, Y. C. Tan, L. L. Tan, N. Li, Y. H. Ng, X. Wen and S. P. Chai, Unravelling charge carrier dynamics in protonated g-C3N4 interfaced with carbon nanodots as cocatalysts toward enhanced photocatalytic $\mathrm{CO} 2$ reduction: $\mathrm{A}$ combined experimental and first-principles DFT study, Nano Res., 2017, 1-24. S. Chandrasekhar, Radiative Transfer, Courier Corporation, 1960.

Y. Boyjoo, M. Ang and V. Pareek, Some aspects of photocatalytic reactor modeling using computational fluid dynamics, Chem. Eng. Sci., 2013, 101, 764-784. V. K. Pareek and A. A. Adesina, Light intensity distribution in a photocatalytic reactor using finite volume, AIChE J., 2004, 50, 1273-1288.

11 K. Seino, J. M. Wagner and F. Bechstedt, Ab initio calculation of optical absorption and reflectivity of $\mathrm{Si}(00$ 1)/SiO2 superlattices with varying interfaces, Appl. Surf. Sci., 2008, 255, 787-789.

12 G. Sgalari, G. Camera-Roda and F. Santarelli, Discrete ordinate method in the analysis of radiative transfer in photocatalytically reacting media, Int. Commun. Heat Mass Transf., 1998, 25, 651-660.

13 R. Acosta-Herazo, J. Monterroza-Romero, M. Á. Mueses, F. Machuca-Martínez and G. Li Puma, Coupling the Six Flux Absorption-Scattering Model to the Henyey-Greenstein scattering phase function: Evaluation and optimization of radiation absorption in solar heterogeneous photoreactors, Chem. Eng. J., 2016, 302, 86-96.

J. Moreira, B. Serrano, A. Ortiz and H. de Lasa, TiO2absorption and scattering coefficients using Monte Carlo method and macroscopic balances in a photo-CREC unit, Chem. Eng. Sci., 2011, 66, 5813-5821. M. Modest, Radiative Heat Transfer, 2003, vol. 53.

16 K. A. Jensen, J. F. Ripoll, A. A. Wray, D. Joseph and M. El $\mathrm{Hafi}$, On various modeling approaches to radiative heat transfer in pool fires, Combust. Flame, 2007, 148, 263-279. D. Joseph, P. Perez, M. El Hafi and B. Cuenot, Discrete Ordinates and Monte Carlo Methods for Radiative Transfer Simulation Applied to Computational Fluid Dynamics Combustion Modeling, J. Heat Transfer, 2009, 131, 052701. A. E. Cassano, C. A. Martin, R. J. Brandi and O. M. Alfano, Photoreactor analysis and design - fundamentals and applications, Ind. Eng. Chem. Res., 1995, 34, 2155-2201.

L. M. Fernández-Ahumada, F. J. Casares, J. Ramírez-Faz and R. López-Luque, Mathematical study of the movement of solar tracking systems based on rational models, Sol. Energy, 2017, 150, 20-29.
M. M. De Macedo, C. E. Ponce Saldias and O. H. Ando Junior, Mathematical Modeling of a Solar Tracker System
Two Axes for Generation Photovoltaics, IEEE Lat. Am. Trans., 2016, 14, 4054-4062. and G. Vella, Estimating Radiant Fields in Flat Heterogeneous Photoreactors by the Six-Flux Model, AIChE J., 2006, 52, 3882. 